\title{
Postoperative lung infection in an immunocompromised older adult patient with lung cancer after oncological surgery: a case report
}

\author{
Jun Luo, Ruiqin Zhou, Linjun Li, Lijun Yao, Cheng Zhang \\ Department of Thoracic and Cardiac Surgery, the First Affiliated Hospital of Chongqing Medical University, Chongqing, China \\ Correspondence to: Cheng Zhang. Department of Thoracic and Cardiac Surgery, the First Affiliated Hospital of Chongqing Medical University, No. 1, \\ Youyi Road, Yuzhong District, Chongqing, China. Email: 376724321@qq.com.
}

\begin{abstract}
China is gradually becoming an aging society with the change in the demographics of its population. This is a concern because older adult patients tend to have weaker immune systems. In particular, in patients with malignancies, both the malignant tumor itself and the surgical treatment can contribute to further impairments in immune function. The weakened immune system in older adult patients increases their susceptibility to postoperative complications as compared to in younger patients. However, treatments for inpatients often ignore the compromised immune status of older adult patients during the perioperative period which could lead poor prognosis through various complications. In the case presented here, the patient was already immunocompromised before surgery; she developed fever and cough after a surgery for lung cancer and was later diagnosed with a pulmonary infection, which led to respiratory failure. The outcome of the patient was fair after aggressive treatment. This case was an epitome of therapy with the increase of lung cancer surgery. Infection may not be universal, but the factors always existed. We aimed to find out the reasons cause immunosuppression which leads to infection during perioperative period through the case. Meanwhile, we offered possible treatment to improve immune function state by targeting these causes.
\end{abstract}

Keywords: Lung cancer; postoperative; immunosuppression; lung infection; case report

Submitted Oct 19, 2021. Accepted for publication Dec 15, 2021.

doi: 10.21037/apm-21-3381

View this article at: https://dx.doi.org/10.21037/apm-21-3381

\section{Introduction}

Lung cancer is the most common cancer worldwide and the leading cause of cancer-related death in China (1). In recent years, the rising incidence and mortality rates of lung cancer have imposed a considerable economic burden on patients and society. According to a 2013 report by the American Cancer Society, the number of newly diagnosed lung cancer ranked second and the number of lung cancer-related deaths ranked first (2). Surgery is currently one of the most effective treatments for lung cancer (3). However, the number of lung cancer patients is rising with the aging of the Chinese population. Older adult patients are at higher risk of postoperative infections due to the decline in organ function, the coexistence of a variety of underlying diseases, a weakened defense against pathogens, prolonged hospital stay, and the need for multiple invasive operations (4).

Postoperative infection can directly lead to the deterioration of patient's condition, increase the risk of death, and worsen the prognosis. In addition, it can further prolong hospital stay, increase hospitalization costs, and thus increase the patient's financial burden. Therefore, controlling postoperative infection in lung cancer patients is highly critical. We found these may due to the perioperative immunosuppression. In our current case, an older adult patient undergoing surgery for lung cancer experienced immunosuppression during the perioperative period; she suffered from a severe lung infection after the surgery, but a good outcome was achieved after aggressive treatments. 
We present the following article in accordance with the CARE reporting checklist (available at https://dx.doi. org/10.21037/apm-21-3381).

\section{Case presentation}

A 71-year-old female patient was admitted to our department on July 15, 2020, due to "the presence of nodule in right lung lower lobe on chest computed tomography (CT) during a health check-up 1 week prior". She had a 4-year history of hypertension and diabetes mellitus; however, no systematic treatment had been offered, and neither blood pressure nor blood glucose was tested. She had no particularly notable personal medical history. Physical examination at admission showed: body temperature $(\mathrm{T})$, $36.6^{\circ} \mathrm{C}$; heart rate $(\mathrm{P}), 78$ beats $/ \mathrm{min}$; respiratory rate $(\mathrm{R})$, 20 breaths/min; and blood pressure (BP), $162 / 71 \mathrm{mmHg}$. Chest computed tomography (CT) revealed an irregular nodule about $1.9 \mathrm{~cm} \times 1.3 \mathrm{~cm}$ in size in the dorsal segment of the lower lobe of the right lung, with a lobulated margin and significant enhancement visible following contrast administration. Lung function test showed mild obstructive lung dysfunction and a slight decline in dispersion function. Color Doppler echocardiography revealed a slightly enlarged left atrium and a decrease in left ventricular compliance. Blood cell analysis noted a white blood cell (WBC) count of $3.93 \times 10^{9} / \mathrm{L}$, a neutrophil-to-lymphocyte ratio (NLR) of $48.6 \%$, a T lymphocyte level of $904 / \mu \mathrm{L}$, a CD4 ${ }^{+} \mathrm{T}$ level of $592 / \mu \mathrm{L}$, and a CD8 ${ }^{+} \mathrm{T}$ level of $246 / \mu \mathrm{L}$. Thus, the patient was immunocompromised preoperatively.

The patient underwent surgical treatment after 1 week of preparations. In the general preparation, blood glucose was controlled with insulin, while blood pressure was controlled with amlodipine. Thymalfasin was administered to improve the patient's immunity. For airway preparation, health promotion was offered, along with endurance training to improve exercise tolerance. The patient underwent videoassisted thoracoscopic right lower lobe segmentectomy + lymph node dissection under general anesthesia. A diagnosis of noninfiltrating adenocarcinoma was confirmed by postoperative pathology. The patient was sent back to the specialist intensive care unit (ICU), where vital sign monitoring showed $\mathrm{T}, 36.7^{\circ} \mathrm{C}$; $\mathrm{P}, 80$ beats/min; BP, $141 / 78 \mathrm{mmHg}$; and $\mathrm{R}$ (ventilator-assisted), 13 breaths/min. The endotracheal tube was routinely removed after the patient awoke. The subsequent treatments included cefuroxime sodium (for preventing infection); ambroxol and acetylcysteine (for dispelling phlegm); metformin and insulin (for controlling blood glucose); amlodipine (for controlling blood pressure); and symptomatic supportive treatments including acid suppression, analgesia, antiemetic treatment, and auxiliary treatments for alleviating cough and sputum production.

On the second postoperative day, the patient complained of palpitation and discomfort, with obvious wheezing, shortness of breath, cough, and excess mucus production. Physical examination showed the body temperature was $37.2{ }^{\circ} \mathrm{C}$. Electrocardiogram (ECG) monitoring showed the heart rate (HR) in atrial fibrillation was $130-180$ beats/min, $\mathrm{R}$ was 25-30 breaths/min, and BP was 168/92 $\mathrm{mmHg}$. Wet rales and wheezing rales could be heard in both lungs. Blood gas analysis revealed that the partial pressure of carbon dioxide $\left(\mathrm{PCO}_{2}\right)$ was $36 \mathrm{mmHg}$, the partial pressure of oxygen $\left(\mathrm{PO}_{2}\right.$ was $60 \mathrm{mmHg}$, the inspired oxygen concentration was $41 \%$, and the oxygenation index was 146 . The possible causes of postoperative atrial fibrillation were considered to be the following: (I) postoperative pulmonary infection, (II) heart failure due to postoperative fluid overload leading, (III) hypertensive heart disease, and/or (IV) electrolyte disorders. However, (I) there was no notable fluid overload, (II) the possibility of heart failure was ruled out based on blood pressure, and (III) blood gas analysis suggested a stable internal environment. Bedside chest radiography and fiberoptic bronchoscopy for aspiration and lavage were performed. The chest radiography suggested that the trachea had shifted towards the affected side and that the right lung was inflamed. Bronchoalveolar lavage showed that the airway mucosa was congested and edematous, with the presence of a large amount of yellowish-white, bloody mucous secretion. Thus, we assumed that the patient had suffered from postoperative pulmonary infection and immediately changed the treatment plan to the following: (I) cefoperazone/sulbactam was used instead to treat the infection, (II) continuous fiberoptic bronchoscopy with bronchial aspiration and bronchoalveolar lavage was performed to remove sputum and promote the reopening of the collapsed alveoli, (III) thymalfasin was used continuously to enhance immunity, (IV) high-dose ambroxol was used to resolve sputum, and (V) high-flow oxygen was administered to maintain oxygenation. The presence of Pseudomonas aeruginosa in the bronchoalveolar lavage (BAL) fluid confirmed our suspicion. Meanwhile, the susceptibility testing of cefoperazone/sulbactam also demonstrated that the pathogen was sensitive to these antibiotics. Testing for the perioperative inflammatory indicators and immune function 

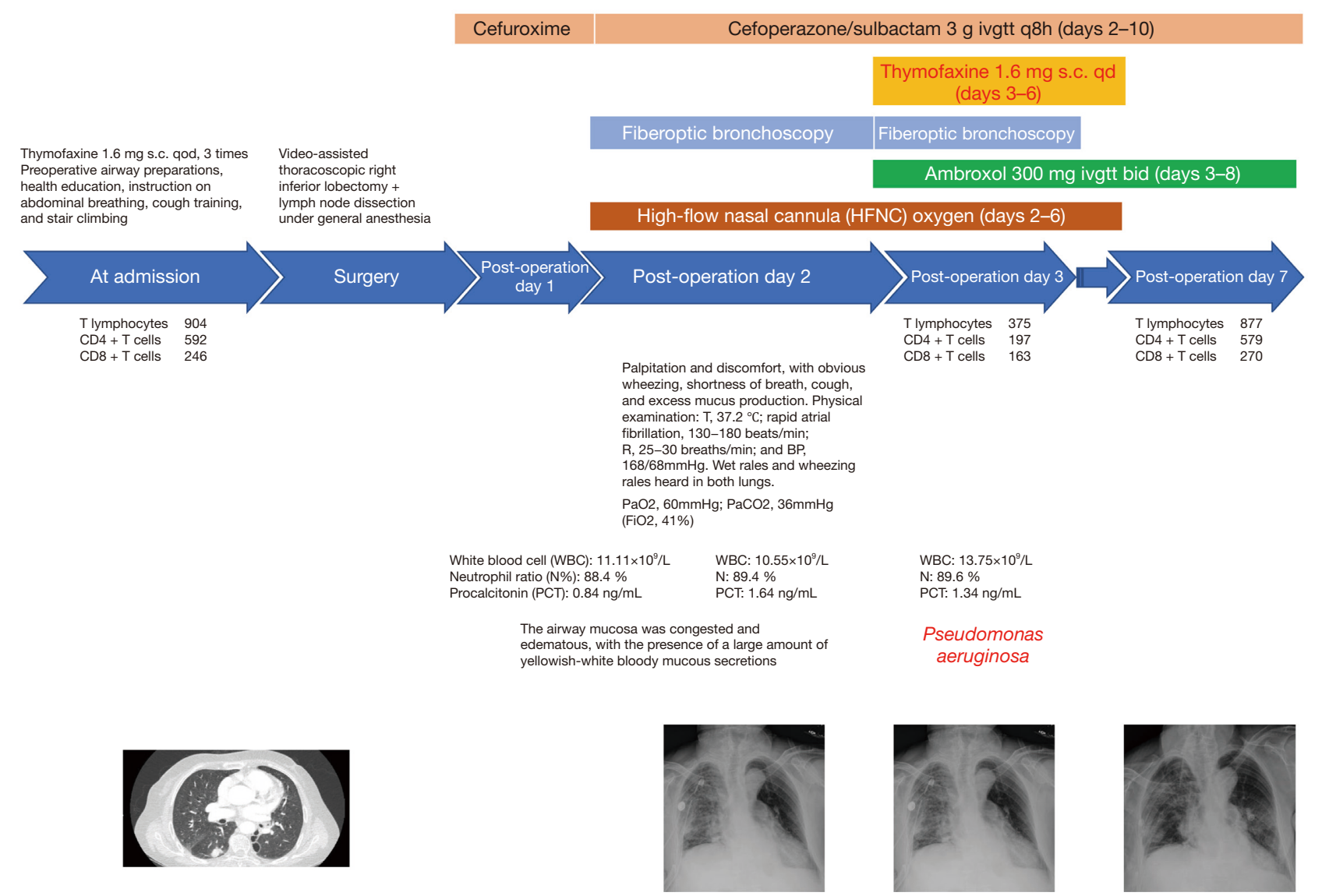

Figure 1 Treatment timeline of the patient. The treatments can be divided into 2 stages: preoperative and postoperative. After admission, the patient was found to be immunocompromised before surgery and was given preoperative preparations to improve immunity. On the second postoperative day, a pulmonary infection developed. On the third postoperative day, immunosuppression was detected; symptomatic treatments such as anti-infection and measures to improve the im-munity level were administered. On the seventh postoperative day, the patient left the intensive care unit. Pictures from left to right are preoperative CT, X-ray of postoperation days 2, 3 and 7 . CT, computed tomography.

revealed that the WBC count fluctuated at $[10-15] \times 10^{9} / \mathrm{L}$, N\% rose to $89-90 \%$ after surgery, and procalcitonin (PCT) levels peaked at $1.64 \mathrm{ng} / \mathrm{mL}$ on the second postoperative day; a second test on the third postoperative day showed a $\mathrm{T}$ lymphocyte level of $375 / \mu \mathrm{L}$, a $\mathrm{CD}^{+} \mathrm{T}$ cell level of $197 / \mu \mathrm{L}$, and a CD8 ${ }^{+} \mathrm{T}$ cell level of $163 / \mu \mathrm{L}$, suggesting the presence of significant postoperative immunosuppression. On the seventh postoperative day, the T lymphocyte count returned close to the preoperative level after the use of thymalfasin. Two days before surgery, the chest radiography suggested that the trachea had shifted towards the affected side and that the right lung was inflamed, suggesting the possibility of pulmonary atelectasis due to sputum retention. The lungs were completely recruited after continuous bronchofiberscopic lavage. The overall condition stabilized after aggressive treatments. The patient was transferred out of the ICU on the seventh postoperative day and was discharged from the hospital on the thirteenth postoperative day. The entire treatment of this patient has been plotted of Timeline figure (Figure 1).

All procedures performed in this study involving human participants were in accordance with the ethical standards of the institutional and national research committees and with the Helsinki Declaration (as revised in 2013). Written informed consent was obtained from the patient for publication of this case report and accompanying images. A copy of the written consent is available for review by the editorial office of this journal. 


\section{Discussion}

Lung infections, respiratory failure, pulmonary atelectasis, heart failure, arrhythmia, empyema, and bronchopleural fistula are common complications after surgical treatment of lung cancer. The incidence of perioperative complications of lung surgery has been reported to be approximately 27\% (5), and it can be even higher in older adult lung cancer patients. In the case presented here, severe lung infection occurred immediately after the surgery. Although the patient finally recovered from the infection, the causes of such lung infection should be actively identified to guide prevention and treatment and thus improve prognosis.

The immune system is an important defense mechanism of the human body and includes cellular immunity and humoral immunity, whose main cellular components are T lymphocytes and B lymphocytes. The body produces an immune response to specific pathogens through a combination of cellular and humoral responses, thus removing or neutralizing pathogens from the body. When the function of any of the defense mechanisms is impaired, recurrent and severe infections are likely to occur and cannot be easily cured. T lymphocytes are involved in mediating cellular immunity and regulating the immune function of the body. CD3 and CD4 antigens are the markers of mature $\mathrm{T}$ lymphocytes and helper $\mathrm{T}$ lymphocytes, respectively, and CD8 is expressed on the surface of cytotoxic $T$ lymphocytes. When an infection occurs, there is a decrease in $\mathrm{CD}^{+}$and $\mathrm{CD}^{+} \mathrm{T}$ lymphocyte population levels and an increase in $\mathrm{CD}^{+} \mathrm{T}$ cytotoxic cell subpopulation levels, resulting in a decline in the $\mathrm{CD}^{+}-$ $\mathrm{CD}^{+}$ratio, and this ratio reflects the function of the cellular immune system.

In our current case, severe immunosuppression persisted throughout the perioperative period, with varying degrees of reduction in total $\mathrm{T}$ lymphocyte count, $\mathrm{CD} 4^{+} \mathrm{T}$ cells, $\mathrm{CD}^{+} \mathrm{T}$ cells, and $\mathrm{CD}^{+}-\mathrm{CD}^{+}$ratio before and after the surgery.

Factors affecting the perioperative immune function can be divided into two main categories: the compromised immune system due to the patients' own conditions and the immunosuppression caused by treatments.

For older adult patients, age is one of the main factors leading to the decrease of perioperative immune function. Aging in humans causes immunosenescence as well as changes in endocrine function and hormonal response levels, leading to a decrease in the regulatory capacity of the immune system. The activity and proliferative capacity of lymphocytes decrease in older adults, resulting in the significant reduction in their functions in generating cytokines and participating in the immune response (6). Lymphocyte and leukocyte mobility and migration also decrease with age, with reduced production of signaling complexes and altered intracellular signaling, leading to lowered cellular function (7). As a result, the immune system in older adults has a weakened ability to adapt to stimuli under stress, predisposing these individuals to immune dysfunction (8) and immunosuppression.

The tumor itself can lead to immunosuppression via multiple mechanisms, including cellular immunosuppression, humoral immunosuppression, and cytokine immunosuppression. Cellular immunosuppression can be contributed by lymphocytes (9), red blood cells, and/ or natural killer $(\mathrm{NK})$ cells and macrophages, respectively. As for humoral immunosuppression, immunoglobulins are significantly decreased in lung cancer patients, leading to a substantially compromised immune system. Cytokines leading to immunosuppression mainly include interleukin 2 (IL-2) (10), interferon-gamma (IFN- $\gamma)(11)$, and tumor necrosis factor (TNF).

Treatments also cause immunosuppression, mainly due to neuroendocrine response and/or surgical factors. The neuroendocrine response is mainly caused by the activation of the hypothalamic-pituitary-adrenal (HPA) axis (12). Physiological and psychological stress leads to increased secretion of catecholamines (including norepinephrine and epinephrine), adrenocorticotropic hormone (ACTH), and cortisol, which can act directly with immune cells, including NK cells, T cells, and macrophages, to suppress cellular immunity, and show an additional effect of indirectly inhibiting cellular immunity by reducing the generation of type 1 cytokines (including IL-12, IFN- $\gamma$, and TNF- $\alpha$ ) via macrophages and helper T cells (13).

The surgical factors of immunosuppression include intraoperative and anesthetic sides. The special intraoperative conditions, including intraoperative blood pressure, blood transfusion, hyperglycemia, hypothermia, and pain, can cause perioperative immunosuppression. In terms of anesthetic factors, general anesthesia modulates the immune system through neurohumoral stress, which inhibits both cellular and humoral immunity to varying degrees; volatile anesthetics (e.g., sevoflurane) inhibit NK cell activity and induce apoptosis of T lymphocytes $(14,15)$; and opioids can also inhibit the function of lymphocytes, macrophages, and NK cells by activating the sympathetic nervous system to release catecholamines (16). 
Thus, perioperative immunosuppression is multifaceted, especially in older adult patients with tumors. In the longterm, immunosuppression may result in an increased risk of tumor recurrence and metastasis. Perioperatively, it can have more immediate consequences, including delayed wound healing and other sepsis events, directly affecting the prognosis.

Therefore, improving the perioperative immune function in older adult patients with tumors is particularly important, and there are three main strategies related to this: (I) improving the active immunity, in which the patients are encouraged to engage in physical exercise during the perioperative period to improve the immune system; (II) enhancing the passive immunity, in which the immune system can be stimulated and regulated by using certain agents, including thymidine $\alpha 1$, interferon, transfer factor, and gamma-globulin, which can increase the activity and number of immune cells a, thus enhancing the body's immune response to stress $(17,18)$; and (III) avoiding immunosuppressive factors, which includes preoperative education to reduce the patient's anxiety and psychological stress, intraoperative use of heaters to maintain stable vital signs, reduction of opioid, and postoperative use of nonsteroidal anti-inflammatory drugs (NSAIDs) for pain relief.

In the case described here, the patient was found to be immunocompromised before surgery, and passive immunotherapy was applied preoperatively to improve the immune status; however, the treatment was not adequate, and the patient's immune level was not retested after the immunotherapy. The patient's immune function was suppressed after she experienced surgical shock; despite the application of passive immunotherapy, lung infection still occurred. Therefore, it may be valuable to assess the immune level in advance and use immune-boosting drugs immediately after surgery, so as to prevent postoperative complications such as lung infection.

In conclusion, older adult oncology patients can experience immunosuppression throughout the perioperative period, and low immune levels may directly lead to an increased risk of perioperative complications and adversely affect the prognosis. For these patients, especially in patients with confirmed preoperative immunosuppression, it is important to test and improve the immune function and actively prevent postoperative complications caused by immunosuppression. When a severe complication (e.g., lung infection in our current case) occurs, aggressive antibiotic use and removal of the infected foci should be offered; in addition, passive immunotherapy (e.g., thymidine $\alpha 1$ ) should be administered to improve the immune level. Both measures will help control the complications and improve the prognosis.

\section{Acknowledgments}

Funding: None.

\section{Footnote}

Reporting Checklist: The authors have completed the CARE reporting checklist. Available at https://dx.doi. org/10.21037/apm-21-3381

Conflicts of Interest: All authors have completed the ICMJE uniform disclosure form (available at https://dx.doi. org/10.21037/apm-21-3381). The authors have no conflicts of interest to declare.

Ethical Statement: The authors are accountable for all aspects of the work in ensuring that questions related to the accuracy or integrity of any part of the work are appropriately investigated and resolved. All procedures performed in this study involving human participants were in accordance with the ethical standards of the institutional and national research committees and with the Helsinki Declaration (as revised in 2013). Written informed consent was obtained from the patient for publication of this case report and accompanying images. A copy of the written consent is available for review by the editorial office of this journal.

Open Access Statement: This is an Open Access article distributed in accordance with the Creative Commons Attribution-NonCommercial-NoDerivs 4.0 International License (CC BY-NC-ND 4.0), which permits the noncommercial replication and distribution of the article with the strict proviso that no changes or edits are made and the original work is properly cited (including links to both the formal publication through the relevant DOI and the license). See: https://creativecommons.org/licenses/by-nc-nd/4.0/.

\section{References}

1. Zhang J, Zhao T, Xu C, et al. Genetic susceptibility of lung cancer in Chinese population: An overview of systematic reviews and meta-analyses. J Evid Based Med 
2017;10:207-11.

2. Siegel R, Naishadham D, Jemal A. Cancer statistics, 2013. CA Cancer J Clin 2013;63:11-30.

3. Mirimanoff RO. Neoadjuvant chemoradiotherapy followed by surgery for stage IIIa and IIIb non-small-cell lung cancer (NSCLC): is it still justified? Chin Clin Oncol 2015;4:49.

4. Gowing SD, Chow SC, Cools-Lartigue JJ, et al. Grampositive pneumonia augments non-small cell lung cancer metastasis via host toll-like receptor 2 activation. Int J Cancer 2017;141:561-71.

5. Ayed AK, Bazerbashi S, Chandrasekaran C, et al. Pulmonary complications following major lung resection for benign and malignant lung diseases. Med Princ Pract 2006;15:114-9.

6. Hirokawa K, Utsuyama M, Kasai M, et al. Aging and immunity. Acta Pathol Jpn 1992;42:537-48.

7. Freidenreich DJ, Volek JS. Immune responses to resistance exercise. Exerc Immunol Rev 2012;18:8-41.

8. Mahbub S, Brubaker AL, Kovacs EJ. Aging of the Innate Immune System: An Update. Curr Immunol Rev 2011;7:104-15.

9. Marino P, Cugno M, Preatoni A, et al. Increased levels of soluble interleukin-2 receptors in serum of patients with lung cancer. Br J Cancer 1990;61:434-5.

10. Nakamura H, Ishiguro K, Mori T. Different immune functions of peripheral blood, regional lymph node, and tumor infiltrating lymphocytes in lung cancer patients. Cancer 1988;62:2489-97.

11. Yano T, Yasumoto K, Togami M, et al. Properties of recombinant interleukin 2-cultured tumor-infiltrating

Cite this article as: Luo J, Zhou R, Li L, Yao L, Zhang C. Postoperative lung infection in an immunocompromised older adult patient with lung cancer after oncological surgery: a case report. Ann Palliat Med 2021;10(12):12894-12899. doi: 10.21037/ apm-21-3381 lymphocytes in human lung cancer. Int J Cancer 1989;43:619-23.

12. Kennedy BC, Hall GM. Neuroendocrine and inflammatory aspects of surgery: do they affect outcome? Acta Anaesthesiol Belg 1999;50:205-9.

13. Ben-Eliyahu $\mathrm{S}$. The promotion of tumor metastasis by surgery and stress: immunological basis and implications for psychoneuroimmunology. Brain Behav Immun 2003;17 Suppl 1:S27-36.

14. Markovic SN, Knight PR, Murasko DM. Inhibition of interferon stimulation of natural killer cell activity in mice anesthetized with halothane or isoflurane. Anesthesiology 1993;78:700-6.

15. Loop T, Dovi-Akue D, Frick M, et al. Volatile anesthetics induce caspase-dependent, mitochondria-mediated apoptosis in human T lymphocytes in vitro. Anesthesiology 2005;102:1147-57.

16. Mellon RD, Bayer BM. Evidence for central opioid receptors in the immunomodulatory effects of morphine: review of potential mechanism(s) of action. J Neuroimmunol 1998;83:19-28.

17. Garaci E, Pica F, Sinibaldi-Vallebona P, et al. Thymosin alpha(1) in combination with cytokines and chemotherapy for the treatment of cancer. Int Immunopharmacol 2003;3:1145-50.

18. Li CL, Zhang T, Saibara T, et al. Thymosin alpha1 accelerates restoration of $\mathrm{T}$ cell-mediated neutralizing antibody response in immunocompromised hosts. Int Immunopharmacol 2002;2:39-46.

(English Language Editor: J. Gary) 